Die Schweiz und die humanitäre Aktion

\title{
Das von Konflikten zerrüttete Afrika : was bei der humanitären Aktion wirklich auf dem Spiel steht
}

\section{Hassan $\mathrm{Ba}$}

\section{(2) OpenEdition \\ 1 Journals}

Electronic version

URL: http://journals.openedition.org/sjep/640

DOI: 10.4000/sjep.640

ISSN: 1663-9677

Publisher

Institut de hautes études internationales et du développement

Printed version

Date of publication: 1 janvier 1999

Number of pages: 85-89

ISSN: 1660-5926

\section{Electronic reference}

Hassan $\mathrm{Ba}$, « Das von Konflikten zerrüttete Afrika : was bei der humanitären Aktion wirklich auf dem Spiel steht », Schweizerisches Jahrbuch für Entwicklungspolitik [Online], 18| 1999, Online erschienen am: 18 Juli 2012, abgerufen am 08 September 2020. URL : http://journals.openedition.org/sjep/640 ; DOI : https://doi.org/10.4000/sjep.640 


\title{
DAS VON KONFLIKTEN ZERRÏTTETE AFRIKA: WAS BEI DER HUMANITÄREN AKTION WIRKLICH AUF DEM SPIEL STEHT
}

\author{
HASSAN BA*
}

A

FRIKA wird heute von Kriegen und Konflikten erschüttert, welche katastrophale humanitäre Folgen, besonders für die anfälligsten Schichten der Gesellschaft haben: Frauen, Kinder, ethnische, religiöse oder kulturelle Minderheiten. Diese Krisen führen auch zu Massenumsiedlungen von Flüchtlingen und Vertriebenen (Ruanda, Burundi, Demokratische Republik Kongo, Sierra Leone, Angola).

Diese neue Situation hat es nicht verfehlt, die internationale Gemeinschaft zu alarmieren, die sich in vielen Fällen durch das Ausmass der Dramen überwältigt, ja sogar unfähig fühlte, zu handeln, um die Opfer zu retten oder zu schützen. Politische Entscheidungsträger der Länder des Nordens begannen, sich Fragen zu stellen und darüberhinaus neue Wege zu ersinnen, um das Instrumentarium und die Mittel der internationalen Zusammenarbeit den jeden Tag komplexer werdenden neuen Krisenkontexten anzupassen.

Gleichwohl ist es wesentlich, über die tugendhaften politischen Aussagen hinauszugehen oder die Grundsatzpetitionen im Licht der historischen Erlebnisse und der grundlegenden Besorgnisse der Empfänger der internationalen Hilfe nachzulesen. Mit anderen Worten, was lehrt uns die konkrete Wirklichkeit des Kivu, Angolas, usw. ? Wie wird die Beziehung zwischen denen, die die Hilfe empfangen, und denen, die sie erteilen, im Alltag erlebt? Was steht bei der Debatte über das Duo « Nothilfe/Entwicklung » für Afrika im wesentlichen auf dem Spiel?

Die obenerwähnten Konflikte sind durch gewisse gemeinsame Punkte gekennzeichnet:

- die Gewaltakteure sind hauptsächlich junge Leute, für die Gewalt eine Lebensart und ein Mittel zur Stärkung ihrer gesellschaftlichen Stellung ist;

口 die «Kriminalisierung» der bewaffneten Gruppen, der Zerfall der Aufstandsbewegungen und die Krise der Staatsgewalt im Inneren;

口 die Verbindung zwischen bestimmten bewaffneten Gruppen und Elementen der «internationalen Kriminalität» in der Phase strategischer Reorganisation in Afrika durch Rauschgifthandel, Waffenschmuggel, Falschgeld- und Rohstoffhandel ;

口 die politisch-militärische Umstrukturierung regionaler Gruppen, die durch die Schwächung des Staates und die Durchlässigkeit der Grenzen ermöglicht wird; 
口 die starke Zunahme der Militärausgaben (Kauf von Waffen, Munition und Ausrüstungen durch die kriegsführenden Parteien).

Diese Konflikte weisen - über ihre gemeinsamen Merkmale hinaus - eine bisher unbekannte Komplexität und Tragweite auf:

- Die von der Charta der Organisation für Afrikanische Einheit (OAU) festgelegte Nichteinmischung in die inneren Angelegenheiten schränkt die Fähigkeit der kollektiven Intervention auf Kontinentsebene ein, wogegen die Staaten einseitig und unbeauftragt in Konflikte militärisch eingreifen (Guinea-Bissau, Demokratische Republik Kongo, Lesotho).

- Das Fehlen eines strukturierten und dynamischen Dialogs zwischen den Forschern, den politischen Entscheidungsträgern und den Führern der Zivilgesellschaft des Kontinents verringert die Möglichkeit, den Ereignissen vorzugreifen und die Konflikte wirksam zu verhüten.

- Das Verschwinden und die Marginalisierung der traditionellen Mechanismen zur Regelung und Bewältigung der Konflikte. Diese Mechanismen betrafen insbesondere die friedliche Verwaltung des Zusammenlebens zwischen Bauern und Viehzüchtern, und die Streitfälle wurden öffentlich geregelt (Palaverbaum), wobei zum Beispiel in Westafrika auf die Sonderbeziehungen zwischen Klans gemäss der Tradition zurückgegriffen wurde.

NDEM man gleichzeitig die verdienstvollen Aktionen der - von den Geberländern unterstützten - humanitären Organisationen würdigt, ist es wichtig, hervorzuheben, dass die hauptsächliche Schwäche ihres Ansatzes in ihrer Beziehung mit dem liegt, was man die «afrikanischen endogenen Kapazitäten» nennen kann. Im Gegensatz zu gewissen Vorurteilen bleiben die afrikanischen Gesellschaften gegenüber den Konflikten, von denen sie zerrissen werden, nicht untätig. Mehrere lokale Akteure greifen ein, um die auftretenden Konflikte zu bewältigen und den Opfern zu Hilfe zu kommen: dies ist bei den Bevölkerungen der Fall, welche die Flüchtlinge und Vertriebenen trotz ihrer äussersten Not aufnehmen; es ist auch bei bestimmten traditionellen Stammesorganisationen in West-afrika, bei Frauenverbänden, nichtstaatlichen Menschenrechtsorganisationen, Bauerngenossenschaften, usw. der Fall.

All diese dynamischen und unternehmenden Kräfte bilden eine neue Zivilgesellschaft. Diese ist in den letzten zehn Jahren entstanden, unter dem Einfluss des Rückzugs und/oder der Schwächung der Staaten, die dem Strukturanpassungsprogramm unterworfen sind; in gewissen Bereichen ist die Zivilgesellschaft auch Erbin der afrikanischen Traditionen der Solidarität und Verantwortung.

Leider haben die internationalen Akteure und die Regierungen des Nordens in zahlreichen humanitären Situationen diesen vielversprechenden Anfängen wenig Bedeutung beigemessen. Schlimmer noch, im Namen der operationellen Effizienz hat man entweder der Instrumentalisierung dieser Akteure oder ihrer Marginalisierung beigewohnt. Die Folgen dieser Situation sind verheerend: das Selbstvertrauen der Bevölkerungen und ihrer Elite ist zerstört. Das Image des Bittsteller-Afrikas verstärkt sich ständig, wodurch einer gewissen Fatalität der Verzweiflung in Afrika selbst Vorschub geleistet wird. 
Die Vielzahl der exogenen Akteure mit verschiedenen, untereinander konkurrierenden Terminkalendern fördert die Aufsplitterung - die Balkanisierung - der lokalen Kapazitäten ; infolgedessen besteht ein wirklicher Gedächtnisbruch zwischen den endogenen Aktionen in den Notsituationen und den in den Friedenssituationen durchgeführten Aktionen. Dieser Bruch führt zu einer Unkenntnis der lokalen institutionellen Errungenschaften, fördert die Verschwendung der Ressourcen und gefährdet die Kontinuität zwischen den verschiedenen Phasen der Krisen.

In der Tat ist das klassische Schema der Differenzierung zwischen Nothilfe/Wiederaufbau/Entwicklung eine Art operationeller Täuschung, die mehr die Unterschiede zwischen den - jeden Tag zunehmend spezialisierten - externen Akteuren als die konkrete Wirklichkeit vor Ort widerspiegelt. Die lokalen Akteure, die in der Notsituation arbeiten, sind oft die gleichen, die in den anderen Phasen tätig sind, mit dem Unterschied, dass sie es je nach den Perioden immer wieder mit verschiedenen Gesprächspartnern zu tun haben.

Woher kommt diese Disqualifizierung der endogenen Akteure? Mehrere Gründe können hierfür angeführt werden :

\ die politische und operationelle Schwäche der lokalen Akteure nimmt ihnen einen Grossteil ihrer Glaubwürdigkeit;

- seitens der externen Akteure zieht die Unkenntnis kultureller Grundlagen, der Traditionen und der soziologischen Entwicklungen Strategie- und Analysefehler nach sich und schadet der nachhaltigen Wirksamkeit der Aktionen; wozu dient in der Tat die Verbreitung der humanitären Normen oder die Verteilung der Reissäcke, wenn die Empfänger nicht die ihrer Würde gebührende volle Achtung erlangen, wenn die Opfer nicht als aktives Subjekt einer kontinuierlichen Geschichte anerkannt werden, wenn die Empfänger von Botschaften sich nicht deren Inhalt wieder aneignen, um sich damit zu identifizieren?

口 Die humanitäre Aktion bleibt in vielen Fällen dem missionarischen und philanthropischen Ideal verhaftet.

\footnotetext{
ÜBER die humanitären Krisen hinaus scheinen grundlegendere Missverständnisse zwischen den politischen Akteuren des Nordens und einem bedeutenden Teil der afrikanischen Elite zu bestehen. Zum ersten : da wo westliche Analytiker in den afrikanischen Konflikten nur menschliche Rückschritte, Bedrohungen für die kollektive Sicherheit und finanzielle Kosten für den Steuerzahler sehen, erkennen und identifizieren afrikanische Verantwortungsträger zuweilen politische Möglichkeiten für wesentliche Veränderungen im Sinne des Aufbaus einer neuen institutionellen Ordnung, die aus einem zwar schmerzlichen, aber dauerhaften Kompromiss zwischen einem geschwächten, seiner Legitimität beraubten postkolonialen Staat und einer dynamischen Zivilgesellschaft hervorgeht, die für die diversen Interessen neuer sozialer Akteure im städtischen und ländlichen Bereich repräsentativ ist. Aus diesem Grunde führt die Tatsache, in allen Fällen - koste es, was es wolle - die Situationen stabilisieren zu wollen, zu einem politischen status quo, der die Entstehung anderer möglicher Zukunftsentwicklungen aufs Spiel setzt.
} 
Zweites Missverständnis : die Hilfe im allgemeinen und die humanitäre Aktion im besonderen treten in Erscheinung, als wären sie rein in ihren Absichten und Interessen, als würden sie sich nicht an interkulturellen und emotionalen Kreuzpunkten abspielen, wobei man vergisst, dass die humanitären Akteure auch auf der Suche nach Bereicherung, Entdeckungen und neuer Bezauberung sind, weil sie aus westlichen Gesellschaften kommen, welche desorientiert, ängstlich und von Technostrukturen überlagert sind. Die Logik der Standardisierung und der bürokratischen Spezialisierung, kurz gesagt, des «humanitären Taylorismus » gefährdet die obersten Tugenden der Geste, nämlich, sich moralisch zu erheben, indem man sich hingibt und anderen gibt.

Im übrigen fühlen sich die Afrikaner im gegenwärtigen Kontext gefangen, wo eine Art Vorherrschaft der Gefühle besteht und fieberhaft am Aufbau der internationalen Zusammenarbeit herumgebastelt wird:

So ist es derzeit Mode, die aus der afrikanischen Zivilgesellschaft hervorgegangenen Eliten zu umgehen und zu versuchen, direkt mit dem «konkreten Volk» mit Hilfe von Kleinstprojekten, Kleinstunternehmen und Kleininitiativen zu arbeiten.

Die jüngsten Beispiele der Konflikte im Gebiet der Grossen Seen zeigen, dass dort, wo eine verantwortliche und würdige politische Elite fehlt, der materielle und institutionelle Aufbau, auch wenn er vom Volk getragen wird, den Wellen der Kriege und der Korruption nicht widerstehen kann.

A FRIKA leidet nicht an einer fehlenden Mobilisierung der Bevölkerung. Es leidet am Fehlen einer neuen politischen Elite, die Träume und Visionen hat und fähig ist, die Erwartungen zu kristallisieren und die notwendigen sozialen Veränderungen hervorzurufen und zu begleiten.

Wie bereits festgestellt, haben die jüngsten « Intellektuellenmoden » die abträgliche Wirkung, dass sie die langsame Tötung der afrikanischen Staaten im Namen des Liberalismus und des Wiederaufkommens der Zivilgesellschaft bewirkt haben. Jedoch bildet die Schwächung der Staaten, vor allem der gesetzlichen und administrativen Gewalt, einen wesentlichen Faktor der Verschlimmerung und Destrukturierung der Konflikte (Chaos, Plünderungen, usw.). Die jüngste Geschichte in Afrika lehrt, dass der Tod des Staates die Kriminalisierung der Wirtschaften und der Konflikte sowie die Privatisierung der Gewalt bedeutet und vor allem die Unfähigkeit der Zivilgesellschaft, in diesem unsicheren Umfeld zu handeln, mit sich bringt. Die grösste Herausforderung, die sich den afrikanischen Verantwortlichen heute stellt, ist die Beantwortrung der Frage: «Wie kann man den Rechtsstaat in Afrika stärken, ihm eine politische und soziale Legitimität (wieder)verleihen, ohne erneut den Typus des allgegenwärtigen, auf den Klientelismus und die Marginalisierung der Zivilgesellschaft gegründeten postkolonialen Staates zu schaffen?»

In einem heiklen Kontext der Erholung und der Sanierung der Volkswirtschaften erwarten die Verantwortungsträger mehr von ihren Partnern : finanzielle Investitionen und Entwicklungshilfe.

Ist Afrika nicht reif, um Unternehmen zu gründen und Arbeitsplätze zu schaffen? Ist es nicht bereit, Produkte in die Märkte des Nordens zu exportieren? Die 
Öffnung der Märkte und der Abbau der Zoll- und Tarifschranken sind die Taten, die von der ersehnten neuen Zusammenarbeit am meisten erwartet werden.

A

BSCHLIESSEND muss nochmals nachdrücklich betont werden, dass für die afrikanischen Verantwortlichen die Zeit gekommen ist, ihre eigene Verantwortung in der ihnen zustossenden Situation festzulegen; sie werden dadurch an politischer Reife und an Glaubwürdigkeit auf der internationalen Bühne gewinnen. Auch muss erneut gesagt werden, dass trotz der spektakulären Krisen die wirkliche, verborgene und anhaltende Tendenz das Wiederaufleben Afrikas ist; zahlreiche Anzeichen bezeugen es: das Wirtschaftswachstum stellt sich nach und nach wieder ein, die Führung der staatlichen Dienste wird transparenter, der Rechtsstaat setzt sich hier und da durch.

Der Ausgang bleibt indes ungewiss. Daher die Dringlichkeit, dieses « Herauskommen aus dem Tunnel » mit der Rehabilitierung und der Förderung einer neuen politischen Generation einhergehen zu lassen, einer Elite, die den auf dem Spiel stehenden Herausforderungen gewachsen ist, im Sinne der Förderung einer wirklichen Partnerschaft zwischen den internationalen und den lokalen Akteuren. Diese Partnerschaft liesse sich durch einige wesentliche Initiativen verwirklichen:

口 Förderung der afrikanischen regionalen, nationalen und lokalen Organisationen, um ihre operationelle Wirksamkeit, ihre Glaubwürdigkeit und ihre Erkennbarkeit zu steigern;

口 Gewährung einer den Bedürfnissen der Partner angepassten institutionellen Unterstützung ;

a Veranstaltung von Ausbildungs- und Abstimmungsworkshops, um einen Erfahrungsaustausch und eine endogene Reflexion über die humanitären Probleme in Afrika zu fördern. 